Editorial

\title{
The Kidney in Familial Mediterranean Fever
}

Familial Mediterranean fever (FMF) is a hereditary periodic fever disease that presents with recurrent attacks of peritonitis, pleuritis, arthritis, or erysipelas-like erythema ${ }^{1}$. The attacks last up to 3 days, with varying degrees of frequency. One of the most devastating complications of the disease is amyloidosis, which primarily affects the kidneys but can involve the liver, intestines, and the heart. Colchicine is the drug of choice for $\mathrm{FMF}^{2,3}$. It controls acute attacks of FMF and fends off development of amyloidosis. Since the introduction of colchicine for treatment of FMF, amyloidosis is infrequently seen among compliant patients.

In the past, the mere presence of proteinuria in a patient with FMF suggested a diagnosis of renal amyloidosis since it was the most common kidney involvement in this disease. Nevertheless, more than 30 years ago, Eliakim, et al already reported that patients with FMF could have renal diseases other than amyloidosis ${ }^{4}$. Among these are hemorrhagic glomerulonephritis, chronic glomerulonephritis, Henoch-Schönlein nephritis, and polyarteritis nodosa (PAN). In another report in which Eliakim, et al studied 106 FMF patients, amyloidosis was found in only 13 of 19 patients with persistent albuminuria ${ }^{5}$. Renal biopsies in 4 of the 6 cases without amyloidosis showed minimal nondiagnostic changes, focal glomerulonephritis, or segmental glomerular sclerosis. Both studies were published prior to the introduction of colchicine treatment. More recently, Said, et al reported kidney biopsy pathologies in 15 patients with FMF and proteinuria ${ }^{6}$. Thirteen had urine protein of more than $1 \mathrm{~g} / 24 \mathrm{~h}$. Only 7 had amyloidosis, while 6 had mesangial proliferative glomerulonephritis (with IgA or IgM deposition), and 2 had rapidly progressive glomerulonephritis. Most of the patients were noncompliant for colchicine.

Owing to the high efficacy of colchicine treatment in preventing amyloidosis and to the possible existence of other renal pathologies, today it seems imperative to perform a kidney biopsy when a patient with FMF presents with proteinuria. This policy raises several questions: When
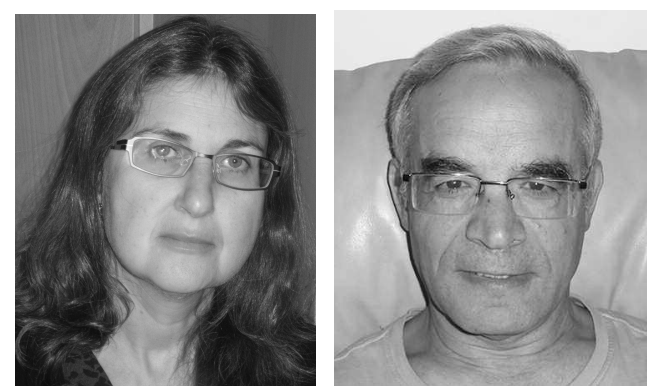

should we perform the biopsy and at which range of proteinuria? Should we perform rectal biopsy prior to kidney biopsy in these patients? Are patients with FMF more susceptible to develop renal diseases (other than amyloidosis) compared with the general population? Do patients with FMF have a more specific type of glomerulonephritis? Does colchicine have a role in non-amyloid kidney disease (NAKD)? Is it possible a priori (before biopsy) to make a diagnosis of kidney disease in patients with FMF? Is urinalysis helpful?

Usually the indication for renal biopsy is either isolated proteinuria of $\geq 1 \mathrm{~g} / 24 \mathrm{~h}$ or when there is active urine sediment, [e.g., presence of dysmorphic red blood cells (RBC) or RBC casts] or in acute renal failure. Kidney biopsy is also indicated when a systemic disease is suspected. It might be argued that in patients with FMF, renal biopsy should be performed even in milder proteinuria in order to diagnose amyloidosis earlier and increase the dose of colchicine. However, these patients should receive a higher dose of colchicine once proteinuria appears, even before performing renal biopsy.

Additional questions arose regarding the necessity of rectal biopsy prior to kidney biopsy in patients with FMF presenting with proteinuria. Rectal biopsy has a diagnostic yield of $75 \%$ to $85 \%$ if an adequate submucosal specimen is obtained ${ }^{7}$. However, it is associated with a risk of bleeding, bacteremia, and patient discomfort. It also requires an endoscopic procedure done by a skilled endoscopist. Yet, in cases of negative results, the patient should have additional biopsy from the kidney. Since the prevalence of renal amyloidosis is expected to lessen with time due to better availability of colchicine and improved compliance, one should look for findings other than amyloid pathology, and rectal biopsy seems unnecessary.

Henoch-Schönlein purpura (HSP) and PAN may affect the kidneys and are more common in patients with FMF. HSP is seen in $2.75 \%$ to $7 \%$ of FMF patients and PAN in $1 \%$ to $2.1 \%$ of patients, whereas in the general population,

See FMF with proteinuria, page 2083

Personal non-commercial use only. The Journal of Rheumatology Copyright (C) 2013. All rights reserved. 
the incidence of HSP is 20:100,000 and of PAN 4-10:1,000,000 ${ }^{8}$. PAN in FMF patients has unique features: It appears at an earlier age than classic PAN, perirenal hematoma appears in up to $50 \%$ of patients, some have overlap features of PAN and microscopic PAN, and the prognosis is relatively good ${ }^{9}$. Other than reports of these 2 diseases, there are no large epidemiological studies suggesting that non-amyloid glomerular disease is more frequent in FMF patients than in the general population.

In the report by Said, $e t a l^{6}$, and in an article by Kukuy, et al in this issue of The Journal ${ }^{10}$, no cases of HSP or PAN were noted, probably because renal biopsy is usually not indicated in patients with HSP and because PAN is rare ${ }^{6,10}$. In these series, types of NAKD were similar to those expected in non-FMF patients, where IgA nephropathy, focal segmental glomerulosclerosis, and mesangial lesions are commonly seen.

Older reports showed that colchicine treatment reduced proteinuria and fibrosis in animal models of nephritis ${ }^{11,12}$. Several case reports showed that FMF patients with non-amyloid renal involvement - including IgG and IgM mesangial proliferative glomerulonephritis and $\operatorname{IgA}$ nephropathy - also responded to colchicine therapy alone ${ }^{13,14}$. This observation suggests that colchicine may suppress the inflammatory process affecting the kidneys. However, data are limited since there are no controlled trials testing the effect of colchicine in NAKD.

The current report by Kukuy, et al describes findings in a retrospective study conducted over 10 years (2001-2011) that included all patients with FMF undergoing kidney biopsy for proteinuria $>0.5 \mathrm{~g} / 24 \mathrm{~h}^{10}$. They found that among 25 FMF patients, 10 (40\%) had NAKD including mainly focal glomerulonephritis and focal segmental glomerular sclerosis. This rate of NAKD is similar to that reported by Said, et $a l^{6}$. Contrary to these results, among 2436 patients from the Turkish FMF study group, 12.9\% had biopsy-proven amyloidosis (amyloid kidney disease, AKD), while only $0.8 \%$ had NAKD ${ }^{15}$. Pathologies reported in these patients included: mesangiocapillary glomerulonephritis, mesangial proliferative glomerulonephritis, and diffuse endocapillary proliferative glomerulonephritis. Thus, the rate of NAKD may differ in various countries or populations due to differences in colchicine availability, compliance, and additional environmental factors.

A unique analysis done in the current report by Kukuy, et $a l$ is a comparison between patients with AKD and those with NAKD ${ }^{10}$. In a multivariate analysis, only severe FMF, hypertension, and proteinuria $>3.5 \mathrm{~g} / 24 \mathrm{~h}$ were more common in the patients with renal amyloidosis. Nevertheless, these findings were not useful in predicting amyloidosis in an individual patient. Urinalysis, too, was not a discerning factor, since active sediment was seen in both groups of patients. Accordingly, a clinical prediction by the treating physician was inadequate and failed to establish a correct diagnosis in 37\% of the patients with AKD and 63\% of the patients with NAKD. Several known factors may increase the risk for amyloidosis in patients with FMF, including elevated serum amyloid A (SAA) serum level, male sex, SAA1 polymorphism, and family history of amyloidosis. These characteristics were not taken into account in the analysis by Kukuy, et al. It would be of interest to learn whether such analysis could have improved the ability to predict the development of renal amyloidosis.

In the study by Kukuy, et al, FMF severity correlated with AKD. On the other hand, severity of FMF correlates with M694V homozygosity ${ }^{1}$. Nevertheless, this genotype was seen in 13/15 (86\%) patients with AKD and in 6/10 (60\%) patients with NAKD, a difference that failed to reach statistical significance probably because of the small size of the groups. In previous studies, $90 \%$ to $100 \%$ of FMF patients with renal amyloidosis or endstage renal disease (ESRD) were homozygous for the M694V or M694I compared to $40 \%$ to $64 \%$ of patients without amyloidosis a difference that was highly significant ${ }^{16,17}$. It still seems that homozygosity for the 694 mutation is a major risk factor for renal amyloidosis.

ESRD was more common in patients with AKD than in patients with NAKD during the study period ( $75 \%$ vs $25 \%)$. This finding is in line with a recent article by Twig, et $a l^{18}$, in which the cause of ESRD in FMF patients was amyloidosis in all the women and in $92 \%$ of the men, who had been followed since 1973 for a mean of 24 years. These data suggest that the main cause of severe renal involvement with renal failure - in FMF - is still amyloidosis; and renal involvement not due to amyloidosis is relatively rare or milder and does not often progress to ESRD.

In summary, there is accumulating data that FMF patients may suffer from both AKD and NAKD. Owing to the improvement in FMF treatment, we will probably see more NAKD than amyloidosis. Since clinical data are not precise enough to diagnose the exact pathology a priori, renal biopsy in FMF patients with proteinuria is imperative, with indications similar to those for non-FMF patients.

\section{HAGIT PELEG, MD, ELDAD BEN-CHETRIT, $M D$,}

Professor of Medicine, Head, Rheumatology Unit, Hadassah-Hebrew University Medical Center, Jerusalem, Israel

Address correspondence to Dr. Ben-Chetrit, Hadassah-Hebrew University Medical Center, Jerusalem, Israel, POB 12000.

E-mail: eldad@hadassah.org.il

\section{REFERENCES}

1. Ben Chetrit E, Levy M. Familial Mediterranean fever. Lancet 1998; 351:659-64.

2. Goldfinger SE. Colchicine for Familial Mediterranean fever. NEJM 1972;287:1302.

Personal non-commercial use only. The Journal of Rheumatology Copyright $\odot$ 2013. All rights reserved. 
3. Ozkan E, Okur O, Ekmekci A, Azcan R, Tay T. A new approach to the treatment of periodic fever. Med Bull Istanbul 1972;5:44-9.

4. Eliakim M, Levy M, Ehrenfeld M. Recurrent polyserositis (familial Mediterranean fever). Amsterdam: Elsevier/North Holland Biomedical, 1981

5. Eliakim M, Rachmilewitz M, Rosenmann E, Niv A. Renal Manifestations in Familial Mediterranean Fever. Isr J Med Sci 1970;6:228-45.

6. Said R, Hamzeh Y, Said S, Tarawneh M, Al-Khateeb M. Spectrum of renal involvement in Familial Mediterranean fever. Kidney Int 1992;414-9.

7. Gafni J, Sahar E. Rectal biopsy for the diagnosis of amyloidosis. Am J Med Sci 1960;240:323-6.

8. Watts RA, Lane SE, Scott DG, Koldingsnes W, Nossent H, Gonzalez-Gay MA, Garcia-Porrua C, Bentham GA. Epidemiology of vasculitis in Europe. Ann Rhem Dis 2001;60:1156-7.

9. Ozen S, Ben-Chetrit E, Bakkaloglu A, Gur H, Tinaztepe K, Calguneri M, et al. Polyarteritis nodosa in Patients with Familial Mediterranean Fever (FMF): a concomitant disease or a feature of FMF? Semin Arthritis Rheum 2001;30:281-7.

10. Kukuy O, Livneh A, Ben David A, Kopolovic J, Volkov A, Shunar Y, et al. Familial Mediterranean fever with proteinuria: clinica features, histology, predictors and prognosis in a cohort of 27 patients. J Rheumatol 2013;40:2083-7.

11. Amore A, Perruzi L, GIanoglio B, Cirina P, Brusa P, Cavallo F, et al. Inhibition of experimental $\operatorname{IgA}$ nephropathy by colchicine. Contrib Nephrol 1995;111:155-60.
12. Milner LS, Lotan D, Millis M, Goodyer PR, Fong JS, Kaplan BS Colchicine reduces proteinuria in passive Heyman nephritis. Nephron 1987;46:11-7.

13. Akpolat T, Akpolat I, Karagoz F, Yilmaz E, Kandemir B, Ozen S. Familial Mediterranean fever and Glomerulonephritis and review of the literature. Rheumatol Int 2004;24:43-5.

14. Ceri M, Unverdi S, Altay M, Yilmaz, Duranay M. An unusual effect of colchicine treatment in Familial Mediterranian Fever-associated glomerulonephritis. Rheumtol Int 2011;31:971-2.

15. Tunca M, Akar S, Onen F, Ozdogan H, Kasnpcopur O, Yalcinkaya F, et al. FMF in Turkey. Results of a nationwide multicenter study. Medicine 2005;84:1-11.

16. Livneh A, Langevitz P, Shinar Y, Zaks N, Kastner DL, Pras M, et al. MEFV Mutation analysis in patients suffering from amyloidosis in Familial Mediterranean Fever. Amyloid 1999;6:1-6.

17. Ben-Chetrit E, Backenroth R. Amyloidosis induced end stage renal disease in FMF patients is highly associated with point mutation in the MEFV gene. Ann Rheum Dis 2001;60:146-9.

18. Twig G, Livenh A, Vivante A, Afek A, Shamiss A, Derazne E, et al. Mortality Risk Factors Associated with Familial Mediterranean fever among a cohort of 1.25 million adolescents. Ann Rheum Dis 2013 March 16.

J Rheumatol 2013;40:1948-50; doi:10.3899/jrheum.131135 